\title{
Reply to the letter "Chronic kidney disease and cognitive impairment in the very old"
}

\author{
Han Wang ${ }^{1,2} \cdot$ Chenli Fang $^{1} \cdot$ Lin Cai $^{1} \cdot$ Birong Dong $^{2} \cdot$ Juelin Deng $^{2}$
}

Published online: 19 February 2016

(C) Springer International Publishing Switzerland 2016

In the newsletter, Dr. Kawada suggested that the eGFR should be cautiously used in the very old. We agree with his approach; in fact, the possibility was outlined in the Discussion. With regard to the second comment, it is consistent with our findings as we also suggested that "the difference in cognitive function between the young old and the oldest old may mainly derive from different ages"; survivors in fact may have different risk factors for cognitive impairment and other diseases.
Compliance with ethical standards

Conflict of interest None.

Ethical approval The Research Ethics Committee of the Sichuan University approved the study.

Informed consent Informed consent was obtained from all subjects.

This comment refers to the article available at doi:10.1007/s40520015-0526-x.

Han Wang

7972348@qq.com

1 Cardiovascular Disease Research Institute, The Third People's Hospital of Chengdu, The Second Affiliated Chengdu Clinical College of Chongqing Medical University, Sichuan, People's Republic of China

2 Department of Geriatrics, West China Hospital University, Sichuan, People's Republic of China 